\title{
Thorax masculinization in a transsexual patient: Inferior pedicle mastectomy without an inverted $\mathrm{T}$
}

\section{scar}

\author{
Adriana Marcela Gonzalez Cely ${ }^{1}$, Carlos Enrique Triana ${ }^{2}$, Lina Maria Triana ${ }^{2}$ \\ ${ }^{1}$ Department of Plastic Surgery, University Industrial of Santander, Bucaramanga; ${ }^{2}$ Department of Plastic Surgery, Corpus and Rostrum \\ Clinic, Cali, Colombia
}

Transsexual individuals with gender dysphoria or gender identity disorder are rare, with a prevalence reported to range from $0.002 \%$ to $0.014 \%$. Studies have shown that mastectomy yields significant improvements in body image and self-esteem in female-to-male transsexual patients. In patients with grade III breast ptosis, mastectomy with a nipple-areolar complex (NAC) graft is the most commonly used technique, although it has several disadvantages. In the case described herein, a bilateral mastectomy preserving the NAC in an inferior pedicle was performed. Additionally, a thin superior thoracic dermal-fat flap was preserved and eventually sutured at the previous inframammary fold, preventing an inverted T scar. This case shows the advantage of this technique for preserving the blood supply and innervation of the NAC, with a low hypopigmentation risk. Furthermore, in this technique, the patch effect does not impair the results of the NAC graft, and there is no need to use an inverted T scar that may result in thoracic feminization.

Keywords Transsexualism / Gender dysphoria / Gynecomastia / Mastectomy

\author{
Correspondence: \\ Adriana Marcela Gonzalez Cely \\ Department of Plastic Surgery, \\ University Industrial of Santander, \\ Carrera 27 Calle 9, Bucaramanga \\ 680001, Colombia \\ Tel: $+57-3158225680$ \\ Fax: +57-6324929 \\ E-mail: adrimar8@gmail.com
}

Received: 27 Jan 2018 • Revised: 16 Apr 2018 • Accepted: 17 May 2018

pISSN: 2234-6163 • elSSN: 2234-6171・ https://doi.org/10.5999/aps.2018.00108・ Arch Plast Surg 2019;46:262-266

\section{INTRODUCTION}

The term transsexual was first used in 1940 after the appearance of several individuals who wanted to live in a permanent way as the opposite sex; this term emerged to describe the newly-identified phenomenon of individuals who are born with one sex and want to belong to another [1]. Today, this phenomenon is described using the terms gender dysphoria or gender identity disorder (GID) [2]. GID is a rare condition, and few studies investigating it have been published [3]. The American Psychiatric Association reported a prevalence in 2003 of $0.005 \%$ to $0.014 \%$ in adult men and $0.002 \%$ to $0.003 \%$ in adult women.
Various studies have shown that patients with gender dysphoria have a negative body image, especially in the thorax. In femaleto-male transsexual patients, mastectomy significantly increases body satisfaction, not only in terms of general appearance, but also through improvements in quality of life and self-esteem [46]. Techniques such as modified breast reduction for cases of gynecomastia [7], as well as transareolar, semicircular, concentric, inferior and superior pedicle, and nipple-areola graft mastectomies have been used [8].

In female-to-male transsexual patients with grade III Regnault breast ptosis, mastectomy with a nipple-areola graft is the most commonly used technique, since it enables quick and simple re- 

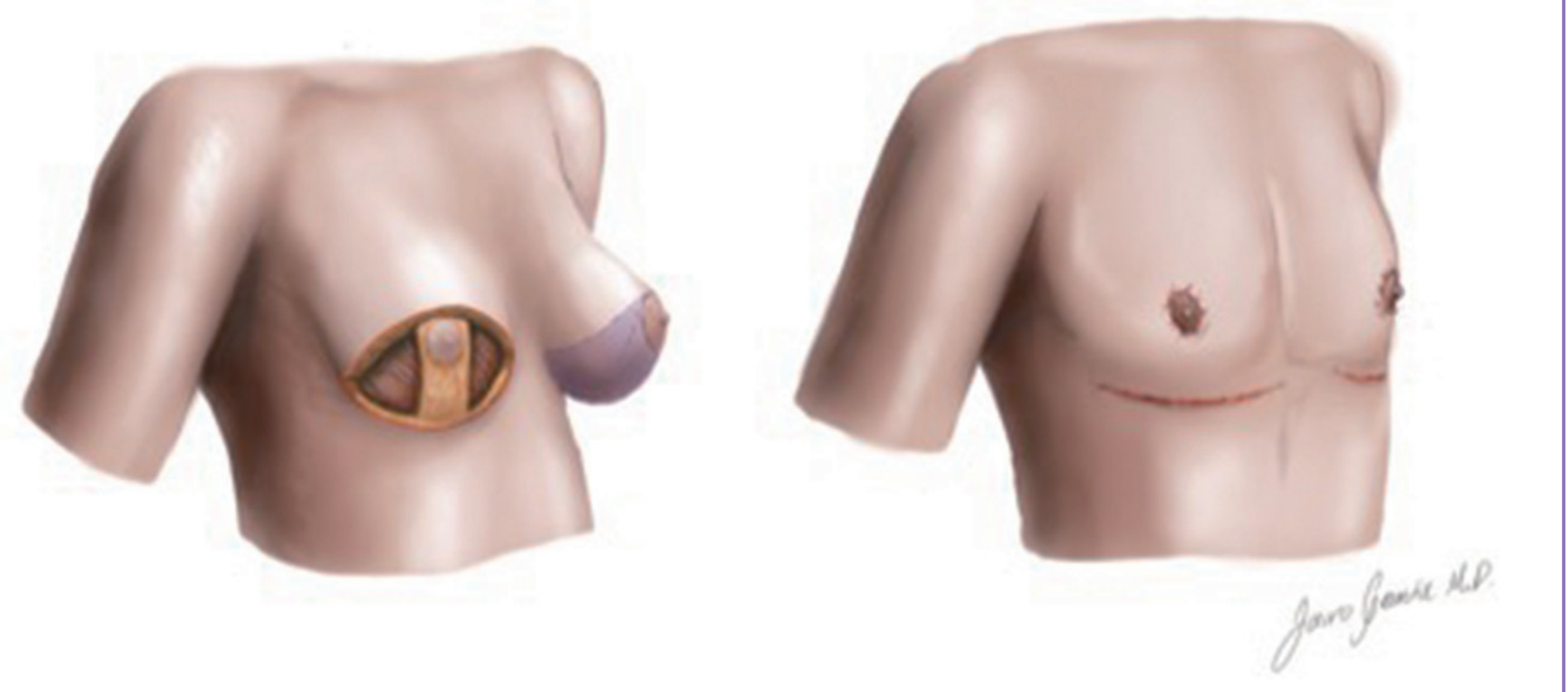

positioning of the nipple-areolar complex (NAC); its disadvantages include loss of sensation, risk of necrosis, hypopigmentation, a long scar, and the patch effect [9].

We present a case where a NAC-preserving mastectomy was performed with an inferior pedicle to keep the neurovascular attachments in place; this technique enabled the preservation of nipple sensitivity, posed a low risk of hypopigmentation, and prevented the patch effect without increasing scarring (Fig. 1).

\section{CASE}

A 27-year-old female-to-male transsexual patient with a diagnosis of gender dysphoria since 2010 presented for a consultation. This individual had been living in a male role since 2005.

Upon physical examination, we observed secondary sexual characteristics, with moderate breast tissue (B-cup bra size), grade III breast ptosis, limited elasticity of the skin, a body mass index of $23.5 \mathrm{~kg} / \mathrm{m}^{2}$, and male characteristics due to hormonal therapy 2 years prior to the consultation. A bilateral mastectomy with NAC preservation was planned, with an inferior pedicle including a skin flap that would not involve a vertical scar. Hormonal therapy was suspended 4 weeks prior to surgery and breast ultrasonography was performed to evaluate the breast prior to surgery, confirming the presence of normal breast tissue. The patient also provided informed consent.

Markings were made with the patient standing up; the inframammary fold was drawn, and the pinch test was used to calculate how much excess skin was present in the inferior pole. An elliptical marking was made in the lower portion of the breast. The skin incision was made on the superior border of the ellipti- cal marking.

A superior thoracic dermal-fat flap was dissected, and its thickness was reduced by removing breast tissue and excess fat. On the skin previously marked with the elliptical shape on the inferior breast pole, an inferior pedicle was marked with a width of 6 $\mathrm{cm}$ and a height extending from the inframammary fold to the NAC. Block resection (skin, breast tissue, and fat) was done on each side of the previously marked inferior pedicle. De-epithelization of the skin of the inferior pedicle with periareolar preservation was done. The dermal-fat thoracic flap was brought down to the location of the inframammary fold. Markings for the new position of the NAC were made $5 \mathrm{~cm}$ lateral to the midsternal line and $4 \mathrm{~cm}$ above the upper border of the resected part, with an areolar diameter of $3.5 \mathrm{~cm}$. The pedicle was sutured to the pectoral fascia with non-absorbable sutures where we previously marked the new NAC position. Resection of a circular portion of skin and subcutaneous tissue over the skin of the thoracic flap was done to expose the NAC and to prevent an inverted $\mathrm{T}$ incision. The thoracic dermal-fat flap was advanced and sutured onto the inframammary fold, with correction of the dog ears. Bilateral drains were used for 5 days postoperatively with a compressive bandage (Fig. 2).

The patient had a good outcome and was satisfied at 1 month postoperatively, with preservation of the vascular bundle of the NAC, progressive improvement of nipple sensation, and a good thoracic contour.

\section{DISCUSSION}

Subcutaneous mastectomy is the first-developed and most com- 


\section{Fig. 2. Thorax masculinization in a transsexual patient}

(A) Preoperative photograph. (B) Right thoracic dermal-fat flap mastectomy. (C) An immediate postoperative photograph. (D) Follow-up photograph 1 month post-surgery.
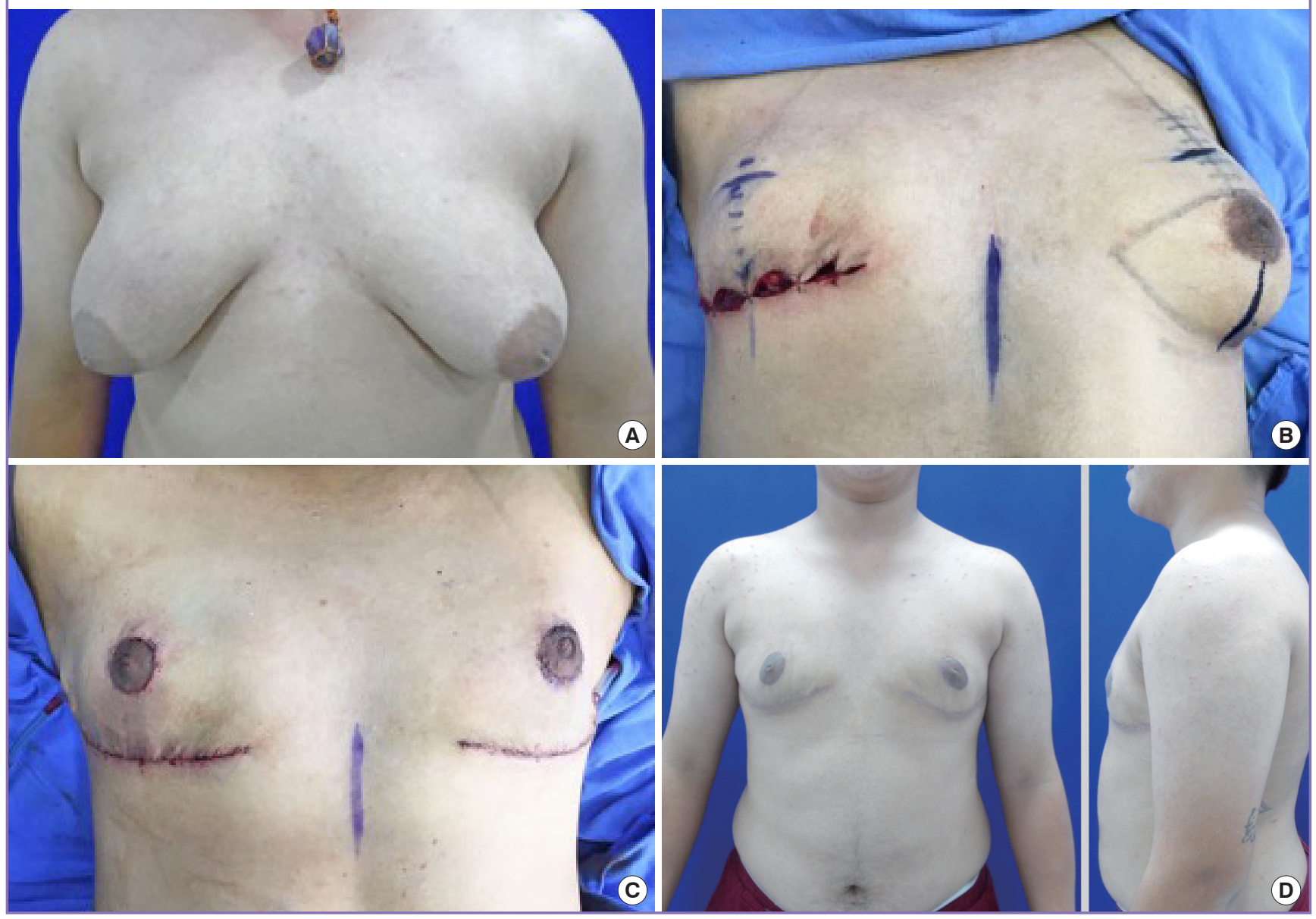

monly used technique for thoracic sex reassignment surgery in patients with female-to-male gender dysphoria. The objective of this technique is to achieve a masculine thorax, and the type of surgery is chosen according to the amount of breast tissue, ptosis, and skin elasticity. Patients with gender dysphoria usually have extensive breast tissue associated with ptosis and non-elastic skin, potentially resulting in large thoracic scars [7].

According to the existing algorithms, in large breasts with grade III ptosis and no skin elasticity, the NAC graft technique is the gold standard $[7,10,11]$. This technique has been shown to be simple and quick. Its disadvantages are loss of sensation; risk of areolar necrosis, which is elevated in smoking patients; hypopigmentation; prolonged healing; the patch effect; and periareolar, inframammary, and mid-thoracic scars [9].

The superior pedicle technique is also used. This method has the advantage of preserving the vascular input of the nipple, but it has the disadvantage of resulting in a visible inverted $\mathrm{T}$ scar with risks of thorax feminization.

Colic and Colic [12] used a circumareolar mastectomy in transsexual patients with large breasts, leaving a superior dermic NAC pedicle, and used round block skin closure; the disadvantages of this procedure were the risk of pedicle necrosis due to a very thin flap, as well as poor skin repositioning when too much excess skin was present, potentially resulting in areolar deformation. Additionally, in cases of grade III gynecomastia with severe ptosis, Cannistra et al. [13] suggested using a periareolar incision with a double areolar pedicle that improved blood supply and nipple sensation.

Thienot et al. [9] described a posterior inferior pedicle similar to the one described in our case study, adding liposuction in some quadrants, that was used in nine patients with grade II gynecomastia or pseudogynecomastia with good results.

The technique used in our case study, similar to the surgical method described by Thienot et al. [9], with the additional use of liposuction, keeps the NAC neurovascular bundles, promoting good sensation and blood supply post-surgery and minimizing the risk of hypopigmentation and the patch effect. Additionally, the dermal-fat flap exposure made it easier to mold the fat 
and breast tissue on the thoracic wall and gave better surgical access, making hemostasis easier. The resultant scarring was similar to the scars described in other techniques, and helped promote a more masculine appearance and made it possible to decrease the areolar size.

The major disadvantage of this technique is that a piece of breast tissue is left as the pedicle, which over the long term can become ptotic, as described by Cardenas-Camarena et al. [14], who recommended an NAC graft for this reason. Nevertheless, we think that decreasing the thickness of the dermal-fat thoracic flap can solve this problem, although irregularities and varying results may occur depending on the overall thoracic texture of the patient. As of the most recent follow-up, our patient has not experienced secondary ptosis and continues to report good satisfaction. We suggest that the technique described in this case study should be further investigated and performed in more patients, in order to evaluate long-term results regarding sensation and the thoracic contour.

In conclusion, for thoracic masculinization in transsexual patients with grade III breast ptosis, a NAC graft mastectomy technique has traditionally been used, although its outcomes have permanent disadvantages. In our case study, a NAC conservation mastectomy with an inferior pedicle technique and the use of liposuction was performed, showing preservation of blood supply and nipple sensation and avoiding the complications secondary to traditional NAC grafts.

Moreover, this technique does not require an inverted $\mathrm{T}$ scar, which can result in a feminine thoracic appearance. This technique provides patients with new treatment options; it is important for patient satisfaction to have minimal scarring and changes in sensitivity. Although thickening of the pedicle area can occur when this technique is used, it can be corrected by degreasing and leaving a thin thoracic dermal-fat flap.

\section{NOTES}

\section{Conflict of interest}

No potential conflict of interest relevant to this article was reported.

\section{Ethical approval}

The study was performed in accordance with the principles of the Declaration of Helsinki. Written informed consent was obtained.

\section{Patient consent}

The patient provided written informed consent for the publication and the use of her images.

\section{Author contribution}

Conceptualization: Gonzalez Cely AM, Triana LM. Data curation: Gonzalez Cely AM, Triana CE. Formal analysis: Triana LM, Gonzalez Cely AM. Methodology, Triana LM, Gonzalez Cely AM. Project administration: Gonzalez Cely AM, Triana LM. Visualization: Triana CE, Gonzalez Cely AM. Writing original draft: Gonzalez Cely AM, Triana LM, Triana CE. Preparation, revision and writing of the manuscript: all authors.

\section{ORCID}

Adriana Marcela Gonzalez Cely https://orcid.org/0000-00024558-1801

Carlos Enrique Triana https://orcid.org/0000-0003-24140107

Lina Maria Triana https://orcid.org/0000-0003-2268-2121

\section{REFERENCES}

1. Sanchez Lorenzo I, Mora Mesa JJ, Oviedo de Lucas O. Psychomedical care in gender identity dysphoria during adolescence. Rev Psiquiatr Salud Ment 2017;10:96-103.

2. Beek TF, Cohen-Kettenis PT, Kreukels BP. Gender incongruence/gender dysphoria and its classification history. Int Rev Psychiatry 2016;28:5-12.

3. Moreno-Perez O, Esteva De Antonio I; Grupo de Identidad y Diferenciacion Sexual de la SEEN (GIDSEEN). Clinical practice guidelines for assessment and treatment of transsexualism. SEEN Identity and Sexual Differentiation Group (GIDSEEN). Endocrinol Nutr 2012;59:367-82.

4. van de Grift TC, Kreukels BP, Elfering L, et al. Body image in transmen: multidimensional measurement and the effects of mastectomy. J Sex Med 2016;13:1778-86.

5. van de Grift TC, Cohen-Kettenis PT, Steensma TD, et al. Body satisfaction and physical appearance in gender dysphoria. Arch Sex Behav 2016;45:575-85.

6. Becker I, Nieder TO, Cerwenka S, et al. Body image in young gender dysphoric adults: a European multi-center study. Arch Sex Behav 2016;45:559-74.

7. Takayanagi S, Nakagawa C. Chest wall contouring for female-to-male transsexuals. Aesthetic Plast Surg 2006;30: 206-12.

8. Monstrey S, Selvaggi G, Ceulemans P, et al. Chest-wall contouring surgery in female-to-male transsexuals: a new algorithm. Plast Reconstr Surg 2008;121:849-59.

9. Thienot S, Bertheuil N, Carloni R, et al. Postero-inferior pedicle surgical technique for the treatment of grade III gynecomastia. Aesthetic Plast Surg 2017;41:531-41.

10. Bonte A. Gynecomastie: techniques chirurgicales et indica- 
tions. EMC Tech Chir Chir Plast Reconstr Esthet 2013;45 (668-D):1-9.

11. Wolter A, Diedrichson J, Scholz T, et al. Sexual reassignment surgery in female-to-male transsexuals: an algorithm for subcutaneous mastectomy. J Plast Reconstr Aesthet Surg 2015;68:184-91.

12. Colic MM, Colic MM. Circumareolar mastectomy in female-to-male transsexuals and large gynecomastias: a per- sonal approach. Aesthetic Plast Surg 2000;24:450-4.

13. Cannistra C, Piedimonte A, Albonico F. Surgical treatment of gynecomastia with severe ptosis: periareolar incision and dermal double areolar pedicle technique. Aesthetic Plast Surg 2009;33:834-7.

14. Cardenas-Camarena L, Dorado C, Guerrero MT, et al. Surgical masculinization of the breast: clinical classification and surgical procedures. Aesthetic Plast Surg 2017;41:507-16. 